\title{
Fotografia digital $x$ analógica: a diferença na qualidade é perceptível?
}

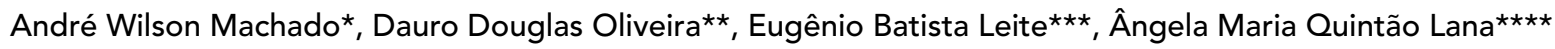

\begin{abstract}
Resumo
A utilização da fotografia digital em Ortodontia tem sido assunto de grande interesse nos últimos anos. Este novo recurso possui inúmeras características que o torna um método de obtenção de imagens muito interessante. Por outro lado, a dúvida quanto à qualidade das imagens gerada por esses novos equipamentos, muitas vezes, desencoraja os profissionais a utilizarem essa nova tecnologia. O objetivo desse trabalho foi avaliar a capacidade de percepção dos ortodontistas em diferenciar as fotografias analógicas das digitais, bem como pesquisar a qualidade das mesmas. Para essa avaliação, foram selecionadas dez fotografias digitais e dez analógicas com um padrão ótimo de qualidade. Todas as tomadas fotográficas foram realizadas pelo mesmo operador. As imagens foram "reveladas" em papel fotográfico e submetidas à avaliação de cinco ortodontistas e cinco alunos de pós-graduação em Ortodontia sorteados aleatoriamente. Os examinadores definiram se as fotografias foram obtidas por câmeras fotográficas analógicas ou digitais e julgaram a qualidade de cada fotografia em uma escala que variava de zero a cinco. Os dados obtidos foram submetidos ao tratamento estatístico e os resultados encontrados demonstraram que a freqüência de erros e acertos ocorreu igualmente nos dois tipos de fotografias ( $p>0,05$ ). Em relação à qualidade das fotografias analisadas, o valor médio conferido pelos examinadores às fotografias analógicas foi de 3,49 e o das digitais foi de 4,07 ( $p<0,001)$. Conclui-se que a qualidade da fotografia digital encontra-se dentro de padrões plenamente aplicáveis à Ortodontia.
\end{abstract}

Palavras-chave: Fotografia. Fotografia digital. Fotografia em Ortodontia.

\section{INTRODUÇÃO}

A utilização da fotografia como rotina na clínica ortodôntica se tornou, ao longo dos anos, um recurso de grande importância para a elaboração do diagnóstico e planejamento ortodôntico, para a avaliação crítica da evolução e resultado dos casos tratados e para auxiliar na comunicação entre profissionais, bem como com os pacientes.
Atualmente, é impossivel falar de fotografia sem se enveredar pela captura digital de imagens. Este novo sistema denominado "Fotografia Digital" é apenas a evolução do método convencional de obtenção de fotografias. Na verdade, as câmeras digitais têm sido utilizadas desde meados da década de 90, porém, estas apresentavam diversas desvantagens como baixa qualidade, alto custo e

* Mestrando em Ortodontia pelo COP - PUC/MG

** Mestre em Ortodontia - Marquette University/EUA.

*** Professor da Disciplina de Fotografia do Mestrado em Ortodontia do COP - PUC/MG

**** Professora Adjunta III da Disciplina de Bioestatística da Escola de Medicina Veterinária da UFMG. 
necessidade de conhecimento técnico específico ${ }^{15}$. Dessa forma, a utilização desse recurso para tomadas fotográficas na clínica ortodôntica era pouco viável ${ }^{8,14}$.

Com a evolução tecnológica das câmeras digitais, a qualidade das imagens geradas melhorou de forma significativa. Além disso, o preço desses equipamentos vem caindo acentuadamente, melhorando a relação custo-benefício ${ }^{6}$.

O emprego desse novo recurso em Odontologia e, especificamente em Ortodontia, tem sido assunto de grande interesse nos últimos anos. A possibilidade da visualização imediata do resultado das fotografias, a eliminação do custo com filmes e sua revelação, além da sistematização do gerenciamento das imagens na clínica têm sido descritas como as principais vantagens da utilização da fotografia digital. Entretanto, como já observado quando da introdução de outras inovações tecnológicas, o uso de fotografias digitais no cotidiano da clínica ortodôntica ainda não é uma realidade. Boa parte dos ortodontistas ainda questionam se a qualidade das fotografias digitais se equivale às obtidas pelas tradicionais câmeras analógicas.

O objetivo desse trabalho foi avaliar a capacidade de percepção dos ortodontistas em diferenciar as fotografias analógicas das digitais, bem como pesquisar a qualidade das mesmas.

\section{REVISÃO DA LITERATURA}

Wilcox e Grimwood ${ }^{20}$ compararam a fotografia digital com a analógica com respeito a dois aspetos. $\mathrm{O}$ primeiro foi em relação à qualidade das imagens proporcionadas e o segundo, a capacidade de se realizar o diagnóstico de lesões dermatológicas a partir das imagens obtidas por ambos os sistemas. Os autores concluíram que, embora a qualidade (reprodução de cor, nitidez, contraste e profundidade de campo) das imagens proporcionadas pela fotografia analógica tenha sido superior, não houve diferença estatisticamente significante entre as fotografias analógicas e digitais para realização do diagnóstico das lesões dermatológicas.

Um estudo comparativo entre a fotografia analógica e a digital foi desenvolvido, em 1999, para avaliar a qualidade das imagens geradas com finalidade de documentação clínica em cirurgia plástica facial ${ }^{19}$. Os autores realizaram fotografias de dez indivíduos com dois equipamentos distintos, um analógico e um digital, com as mesmas condições de luminosidade. Após a avaliação das imagens, foi concluído que, embora ambos sistemas de obtenção de imagens possibilitaram uma completa avaliação pré e pósoperatória dos casos, a reprodução de detalhes e definição das fotografias analógicas apresentaram-se levemente superiores às digitais.

Bock $^{5}$ avaliou a qualidade de fotografias obtidas com câmeras do mesmo fabricante, com características ópticas semelhantes, porém um equipamento era analógico (Canon EOS 30) e outro digital (Canon D30). O autor concluiu que embora a fotografia analógica tenha superado a digital no quesito qualidade, as impressões em papel fotográfico de ambas ficaram bastante semelhantes.

Em relação à comparação das fotografias analógicas e digitais com finalidade ortodôntica, Vilani et al. ${ }^{18}$ selecionaram cinco fotografias: duas extrabucais (frontal e perfil) e três intrabucais (frontal, lado direito e oclusal superior) obtidas com um equipamento analógico e outro digital. Os autores concluíram que embora a qualidade da imagem convencional tenha sido superior, a fotografia digital, para fins de documentação ortodôntica, mostrou-se aceitável.

A revista americana Popular Photography \& Imaging ${ }^{11}$, comparou o sistema de obtenção de imagens digital e analógico, sem considerar marcas e modelos de equipamentos, em relação ao manuseio, praticidade e qualidade final das imagens. Os autores observaram que a qualidade proporcionada pelos equipamentos foi diferente, porém, em muitas situações não foi possível distinguir as 
imagens obtidas por uma câmera digital daquelas registradas pelo equipamento analógico. Concluiuse que o melhor sistema seria aquele que mais se adaptasse às necessidades pessoais e profissionais de cada indivíduo.

Apesar do potencial de aplicação da fotografia digital na Ortodontia, ainda não existem trabalhos comparando a qualidade dessas imagens em relação às analógicas. Portanto, os autores se propuseram a realizar este trabalho com o objetivo de avaliar o estado atual da qualidade das fotografias digitais em Ortodontia.

\section{MATERIAL E MÉTODO}

Os autores selecionaram vinte fotografias intrabucais de pacientes do Mestrado em Ortodontia do Centro de Odontologia e Pesquisa da Pontifícia Universidade Católica de Minas Gerais (COP/PUC-MG), em diferentes fases do tratamento ortodôntico. As imagens selecionadas foram: dez analógicas (em slide de $35 \mathrm{~mm}$ ) e dez digitais, sendo quatro frontais, quatro laterais e duas oclusais com espelho. Todas as tomadas fotográficas foram realizadas pelo mesmo operador. As imagens digitais foram obtidas com uma câmera fotográfica digital semiprofissional, modelo Olympus C-4040 (Olympus Inc., Melville, NY, USA) associada a um difusor de flash da PhotoMed International (Van Nuys, CA, USA). Por outro lado, as imagens analógicas foram adquiridas com uma câmera analógica profissional (monorreflex de 35mm) Canon Rebel 2000 com flash circular Canon ML-3 e objetiva macro 100 Canon (Canon Inc., Taiwan). O filme fotográfico positivo (slide ou cromo) utilizado foi o Kodak Ektachrome 100 (Manaus, AM). Todas as fotografias selecionadas para esse estudo apresentavam um padrão de qualidade excelente de acordo com o critério estabelecido pelo American Board of Orthodontics ${ }^{4}$ (Fig. 1).

Com o objetivo de padronizar a avaliação, todas as imagens foram "reveladas" com resolução de 400 DPI (dots per inch, ou pontos por po- legada) em laboratório digital especializado, por meio de um equipamento profissional, modelo Noritsu 2901 (Noritsu do Brasil S/A, Manaus, $\mathrm{AM}$ ), onde os funcionários da empresa desconheciam a finalidade das imagens. O processo de revelação digital já está em operação em diversas cidades do país, com grande sucesso. Nesse método, as imagens digitais não são impressas como com a utilização de uma impressora a base de jato tinta. As máquinas reveladoras "lêem" os arquivos digitais, convertem as informações em sistema de códigos binários compostos pelos números 0 e 1 , e um sistema a laser sensibiliza o papel fotográfico convencional que então é processado quimicamente ${ }^{17}$.

As fotografias digitais foram obtidas com resolução de 4 Megapixels no formato JPEG (Joint Photographic Experts Group) e nenhum tipo de manipulação foi realizado. Entretanto, as analógicas, em slide de $35 \mathrm{~mm}$, foram inicialmente digitalizadas com resolução compatível com a finalidade desejada (resolução de 400 DPI e escala de saída de $10 \times 15 \mathrm{~cm}$ ), por meio de um scanner profissional, presente no equipamento descrito anteriormente (Noritsu 2901, Noritsu do Brasil S/A, Manaus, AM). O papel utilizado foi o Kodak Edge Generations (Kodak do Brasil, Manaus, AM) que apresentava qualidade fotográfica e tamanho padronizado de $10 \times 15 \mathrm{~cm}$. O processo de transformação de um filme fotográfico convencional (positivo ou negativo) em imagem digital já é uma realidade e diversos laboratórios especializados no país já utilizam essa metodologia como rotina.

É importante diferenciar a terminologia "fotografia analógica", "fotografia digital" e "imagem digital". Quando uma fotografia analógica é digitalizada, esta é convertida em uma imagem digital e não em uma fotografia digital. Pois, esta última é somente um tipo de imagem digital que é adquirida com o uso de câmeras fotográficas digitais.

As imagens reveladas foram organizadas 

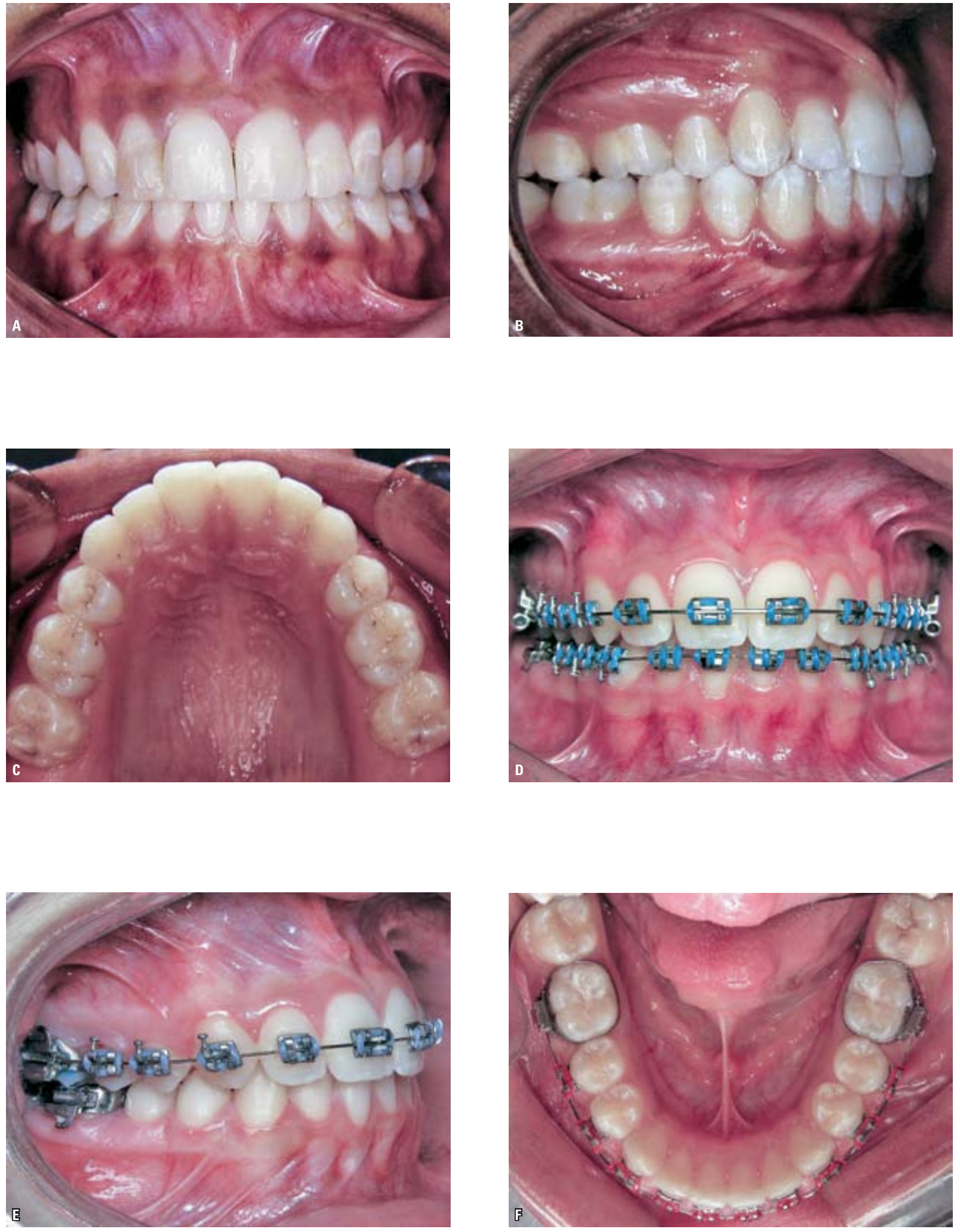

FIGURA 1 - Exemplo de fotografias selecionadas nesse estudo. 
aleatoriamente em um álbum fotográfico, possibilitando a avaliação individualizada de cada fotografia separadamente por cinco professores e cinco alunos do Mestrado em Ortodontia do COP/PUC-MG, sorteados aleatoriamente, e que desconheciam a metodologia aplicada. Os indivíduos selecionados tiveram 10 minutos para avaliar as 20 fotografias e definir se elas foram obtidas por câmeras fotográficas analógicas ou digitais. Além disso, os avaliadores julgaram a qualidade de cada fotografia em uma escala que variava da nota mínima zero (qualidade péssima) a nota máxima cinco (excelente).

Os dados obtidos foram submetidos ao tratamento estatístico. Utilizou-se a tabela de contingência (Qui-quadrado) para verificar as possíveis associações entre a capacidade de percepção com os tipos de fotografias e as categorias de avaliadores. Para a análise da qualidade das fotografias digital e analógica foi utilizado o teste não paramétrico de Wilcoxon. Em relação ao teste do Qui-quadrado e o teste de Wilcoxon foram utilizados os programas estatísticos BioEstat 2.0 e o Saeg 8.0 (Sistema de análises estatísticas) ${ }^{13}$, respectivamente.

\section{RESULTADOS}

Observa-se na tabela 1 que não houve associação entre a taxa de acertos e erros para as fotografias analógicas e digitais, ou seja, a freqüência de erros e acertos ocorreu igualmente nos dois tipos de fotografias, com nível de significância de $5 \%(\mathrm{p}>0,05)$. Pode-se verificar que a quantidade de acertos para as fotografias analógicas foi de 42 e para as digitais foi de 44, enquanto a quantidade de erros foi de 58 e 56, respectivamente.

Foi encontrada associação entre os avaliadores e as taxas de acertos e erros, sendo que a taxa de acertos de alunos foi maior do que a dos professores, com nível de confiabilidade de 95\%. Em outras palavras, a capacidade de diferenciação das fotografias foi maior pelos alunos do que pelos professores (Tab. 2).

Quanto à qualidade das imagens analisadas, o valor médio conferido pelos examinadores para as analógicas foi de 3,49 e das digitais foi de 4,07. Desta forma, a qualidade da fotografia digital apresentou-se superior à analógica, com nível de significância de $0,1 \%$.

\section{DISCUSSÃO}

A utilização da fotografia digital tem despertado grande interesse por parte dos ortodontistas. Porém, a dúvida quanto à qualidade das imagens geradas por esses equipamentos torna o processo de transição da fotografia analógica para a digital difícil e duvidoso. Dentre as principais causas responsáveis pelo descrédito em relação à qualidade das fotografias digitais pode-se citar: 1) a velocidade com que a indústria da imagem digital se desenvolveu resultou em uma ampla diversidade de câmeras fotográficas digitais disponíveis no mercado, o que tornou o processo de escolha mais acertada deste equipamento, para realização de fotografias clínicas em Ortodontia, difícil e duvidosa; 2) a falta de informação e conseqüente dificuldade enfrentada por boa parte dos profissionais para manusear de forma correta esses novos equipamentos; 3) a resistência de alguns profissionais em aceitar a introdução de inovações tecnológicas; 4) o fato da grande maioria das câmeras não ser produzida com finalidade odontológica, pois os fabricantes não estão interessa-

\begin{tabular}{l}
$\begin{array}{l}\text { Tabela 1 - Associação entre a capacidade de percepção } \\
\text { do avaliador e o tipo de fotografia. }\end{array}$ \\
\hline Fotografia \\
\hline
\end{tabular}


Tabela 2 - Associação entre a capacidade de percepção e a categoria do avaliador.

\begin{tabular}{|c|c|c|}
\hline Categoria & \multicolumn{2}{|c|}{ Capacidade de percepção } \\
\hline & Acerto & Erro \\
\hline Professor & 36 & 64 \\
\hline Aluno & 52 & 48 \\
\hline
\end{tabular}

$X^{2}=5,195$

$(\mathrm{P}<0,05)$

dos em um grupo de consumidores tão restrito e 5) a escassez de artigos científicos publicados sobre esse tema. Os poucos estudos encontrados utilizaram equipamentos inadequados, o que pode ter mascarado os dados disponíveis sobre o estado atual da qualidade das imagens digitais.

Após analisar os resultados apresentados na tabela 1, observa-se que não foi possível diferenciar as fotografias analógicas das digitais. Dentre um total de duzentas (200) avaliações (vinte fotografias para dez avaliadores), em somente 86 obteve-se acerto (43\%), enquanto que em 114 (57\%) obtiveram-se erros ( $p>0,05)$. Embora não existam trabalhos semelhantes, específicos para a Ortodontia, Bock ${ }^{5}$ observou que imagens impressas em papel fotográfico de ambos os sistemas demonstraram qualidades bastante semelhantes. Da mesma forma, Popular Photography \& Imaging ${ }^{11}$ observaram que em muitas situações não foi possivel distinguir imagens obtidas por uma câmera digital daquelas registradas por equipamentos analógicos. É importante salientar que esses trabalhos foram realizados por fotógrafos, o que demonstra que a sensibilidade por parte desses profissionais, em diferenciar as imagens é maior do que a dos ortodontistas.

Em relação à diferença entre as categorias de avaliadores (alunos e professores), observou-se que a capacidade de diferenciação das fotografias foi maior pelos alunos do que pelos professores (Tab. 2), com nível de confiabilidade de $95 \%$. Dentre um total de 100 avaliações (vinte fotografias para cinco avaliadores), os professores obtiveram êxito em 36, enquanto os alunos acertaram 52. Embora seja difícil explicar tais resultados, a condição dos alunos ter conseguido maior índice de acertos, pode estar relacionado ao fato destes estarem em contato mais direto e freqüente com as tecnologias digitais. A necessidade dos alunos em trabalhar rotineiramente com ambos sistemas é uma realidade, pois, muitas vezes é necessário utilizar a fotografia analógica para a obtenção de imagens na clínica e a digitalização destas para a preparação de apresentações no Microsoft ${ }^{\circledR}$ PowerPoint ${ }^{\circledR}$ (Redmond, WA, USA). Além disso, antigamente, os cursos de pós-graduação freqüentados pelos professores utilizavam somente a fotografia analógica, pois a digital ainda não existia, o que atualmente já é uma realidade em diversos cursos. De fato, a sensibilização dos alunos pela informática, em geral, parece ser maior do que a dos professores. A exemplo disso, a elaboração das monografias, dissertações e teses por parte dos professores, antigamente, era realizada com a utilização de máquinas de escrever, em detrimento dos computadores. Atualmente, embora o contato com a informática e a Internet (World Wide Web) desde criança, ainda não seja uma realidade, tem aumentado a cada dia.

Fato que até certo ponto surpreendeu os autores foi a melhor qualidade das fotografias digitais. A média das notas conferidas às fotos analógicas foi de 3,49 e das digitais 4,07, com nível de significância de $0,01 \%$. Esse resultado entra em contradição com outros encontrados na literatura $^{5,18-20}$. Dentre esses trabalhos, Vilani et al. ${ }^{18}$, Wall et al. ${ }^{19}$, Wilcox e Grimwood ${ }^{20}$ realizaram a comparação da fotografia analógica com a digital, analisando as primeiras em projetores de slide e as últimas em telas de computadores ou ambas em monitores. O emprego dessa metodologia pode ser questionado, pois além da qualidade das telas dos computadores proporcionar nitidez bastante inferior em relação aos projetores de slide, 
o fato dos avaliadores terem conhecimento prévio do tipo de imagem que estava sendo analisada pode induzir ao tipo de nota associada a cada fotografia.

A resolução das telas dos computadores varia em média de 72 a 90 DPI, ou seja, existem de 72 a 90 pontos em cada polegada linear da imagem $^{1,10,12,16}$. Como a capacidade do olho humano é de enxergar aproximadamente 10 linhas por milímetro, ou seja, distinguir objetos separados em um intervalo de $0.1 \mathrm{~mm}$ a uma distância de $25 \mathrm{~cm}^{3,19}$, isto representa cerca de 300 DPI, ou 300 pontos em cada polegada linear da imagem. Portanto, a utilização de monitores para a avaliação da qualidade de imagens não seria ideal, pois o olho humano facilmente detectaria a presença dos pontos na imagem, diminuindo assim a sua nitidez e definição.

Por outro lado, a metodologia proposta neste trabalho utiliza a mesma resolução para a revelação das fotografias obtidas por ambos sistemas. Desta forma, foi utilizada a resolução de 4 Megapixels (4 milhões de pixels ou de pontos) para a obtenção das fotografias digitais e a resolução de 400 DPI com escala condizente com o tamanho de $10 \times 15 \mathrm{~cm}$ para a digitalização das fotografias analógicas em slides de $35 \mathrm{~mm}$. Em ambos os casos, foi possível obter imagens reveladas em papel fotográfico com resolução de 400 DPI, o que possibilita a obtenção de imagens com alta qualidade. Pois, a resolução mínima necessária para a obtenção de tais imagens seria de 300 DPI, o que estaria condizente com a nossa acuidade visual $^{1,2,7,10,12,17}$. Ao se efetivar uma comparação direta entre a resolução (quantidade de pixels) de um slide de $35 \mathrm{~mm}$ e de uma fotografia digital, obtemos uma diferença notável. Em um diapositivo $35 \mathrm{~mm}$ tradicional existe, em média, de 25 a 30 milhões de pixels de informação ${ }^{14}$. Por outro lado, as câmeras digitais comuns, como a utilizada nesse trabalho, possuem em média de 3 a 6 milhões de pixels. Porém, esta expressiva diferença só será utilizada se grandes ampliações forem realizadas. Em outras palavras, tanto a resolução de 4 Megapixels das fotografias digitais utilizadas nesse trabalho, quanto os 25 Megapixels dos slides terão qualidade similar em um tamanho de 10 X $15 \mathrm{~cm}$. Como o tamanho de imagem utilizado pela maioria das nossas necessidades ortodônticas de rotina, geralmente, não ultrapassa o tamanho de 10 X $15 \mathrm{~cm}$, como por exemplo, impressão de relatórios, confecção de banners, revelação em laboratórios especializados e utilização em publicações científicas, estas resoluções encontravam-se compativeis. Além disso, o fato de ambas as imagens terem sido avaliadas em papel padronizado, e os examinadores as terem associado às notas sem ter conhecimento prévio da origem das imagens (analógicas ou digitais) tornou o processo comparativo mais confiável.

Em contrapartida, pôde-se observar que a qualidade final das fotografias analógicas reveladas em papel fotográfico foi menor que os originais em slides de $35 \mathrm{~mm}$. Desta forma, concluiuse que, embora a digitalização das fotografias analógicas tenha sido realizada em laboratório especializado, com um scanner profissional (Noritsu 2901, Noritsu do Brasil S/A, Manaus, AM) e altas resoluções, as características originais não foram completamente mantidas, o que está de acordo com Lopes et al. ${ }^{9}$ Este achado ressalta uma das vantagens com a utilização das fotografias digitais, pois como essas já consistem de arquivos digitais podem ser facilmente utilizadas em apresentações no Microsoft ${ }^{\circledR}$ PowerPoint ${ }^{\circledR}$ (Redmond, WA, USA) com recurso de multimídia sem nenhuma perda de qualidade, contrariamente, as fotografias analógicas necessitam ser digitalizadas, processo que na maioria das vezes diminui sensivelmente a qualidade do material original ${ }^{9}$. Talvez, se ao invés de se utilizar filmes fotográficos positivos (slides ou cromos), filmes negativos fossem usados, eliminando o processo de digitalização com um scanner, este inconveniente pudesse ser eliminado. Por outro lado, filmes negativos precisam de ampliações, além de 
utilizar um processo de revelação químico mais sensivel, o que também poderia influenciar a qualidade final das fotografias. Na verdade, o que acontece atualmente é que a digitalização dos filmes fotográficos convencionais vem se tornando inevitável. Pois, grande parte dos laboratórios fotográficos não mais utilizam o "antigo" processo químico de revelação analógica à base de substâncias reveladoras e fixadoras, mas sim, os filmes fotográficos convencionais são digitalizados (transformados em "imagens digitais" e não em "fotografias digitais") para, então serem revelados pelo processo digital. Resumindo, a imagem original ou fonte é a "fotografia analógica", porém o método de revelação é o digital.

Outro fator que pode ter influenciado a qualidade das imagens foi a técnica para a obtenção das fotografias. Como todas as tomadas fotográficas foram realizadas por somente um dos autores, e este tem maior prática com câmeras digitais e conseqüentemente maior facilidade de manuseio, o resultado das fotografias digitais talvez tenha sido favorecido. Por outro lado, o equipamento digital utilizado neste trabalho foi uma câmera fotográfica semi-profissional que, embora não seja o equipamento ideal para a realização de fotografias clínicas em Ortodontia, obteve resultados bastante favoráveis, o que não aconteceu com os trabalhos de Vilani et al. ${ }^{18}$, Wall et al. ${ }^{19}$, Wilcox e Grimwood ${ }^{20}$. Enquanto o equipamento analógico foi uma câmera profissional, com melhor indicação para fotografias clínicas do que a digital. Enfim, o trabalho realizado sugere que a fotografia digital pode ser uma alternativa viável e de qualidade para a realização de fotografias clínicas em Ortodontia. Entretanto, com o objetivo único de comparar a qualidade da fotografia digital com a analógica, seria necessária a utilização de duas câmeras (uma analógica e outra digital) profissionais com características semelhantes, associadas à mesma objetiva e ao mesmo tipo de flash, o que será foco de um próximo artigo.

\section{CONCLUSÃO}

Atualmente a utilização da Fotografia Digital, tem gerado grande interesse entre os ortodontistas. Porém, as dúvidas quanto à qualidade gerada por esses novos equipamentos e à viabilidade ou não dessa nova tecnologia, fazem com que grande parte dos ortodontistas ainda não a utilize como rotina na clínica ortodôntica.

Os resultados desse trabalho mostram que após a avaliação de vinte fotografias (dez analógicas e dez digitais), por 5 professores de Ortodontia e 5 alunos de pós-graduação, não foi possivel distinguir as fotografias obtidas por meio de uma câmera fotográfica digital daquelas obtidas por uma analógica ( $p>0,05)$, ou seja, a diferença não foi perceptível.

Em relação à qualidade das fotografias, observou-se que a média das notas das digitais $(4,07)$ foi levemente maior do que as analógicas $(3,49)$ em uma escala de 0 a 5 , com nível se significância de $0,1 \%$. Desta forma, pode-se concluir que embora esses resultados não demonstrem a superioridade da qualidade das imagens obtidas por um sistema em relação ao outro, devido aos fatores discutidos anteriormente, a qualidade da fotografia digital e a viabilidade deste recurso, desde que equipamentos adequados sejam utilizados, encontra-se plenamente aplicável para aqueles profissionais que tenham interesse em lançar mão desta nova tecnologia. 


\title{
Film vs. digital photography: is the difference noticeable?
}

\begin{abstract}
The interest in applying digital photography in orthodontics has increased significantly in recent years. This system presents some properties that make it an interesting alternative to the traditional analogic cameras routinely used in the orthodontic field. However, a number of professionals still question if the digital images quality is comparable to that obtained with analogic photos. Therefore, some orthodontists have not incorporated digital photography to acquire clinical images in their offices. The purpose of this study was to compare the quality of digital and analogical orthodontic photographs and evaluate the ability of the clinicians to differentiate between both types of images. The sample consisted of twenty photographs (ten digital and ten analogical) that followed the American Board of Orthodontists standards. All pictures were taken by the same operator. They were printed in professional photographic paper and submited for evaluation by 5 Orthodontics' professors and an equal amount of orthodontic residents. The evaluators were asked to determine if the images were obtained by digital or analogical cameras, and to grade the quality of each photograph in a zero to five scale. The data recorded was submitted to statistical tests and the results showed no significant diferences in the frequency of errors in ideintifying both types of photographs $(p>0,05)$. The mean quality value for digital photos was 4.07 and 3.49 for analogic images $(p<0,001)$. The results of this study indicate that the quality of digital photography is within the standards required for their application to clinical orthodontics.
\end{abstract}

Key words: Photography. Digital photography. Photography in Orthodontics.

\section{REFERÊNCIAS}

1. ABELSON, M. N. Digital imaging update. Am J Orthod Dentofacial Orthop, St. Louis, v. 116, no. 5, p. 587-590, Nov. 1999.

2. ABELSON, M. N. Parameters for digital imaging. Part 2. Am J Orthod Dentofacial Orthop, St. Louis, v. 118, no. 6, p. 707711, Dec. 2000.

3. ALT, P. M.; NODA, K. Increasing display information content: an introduction. Disponível em: <http://www.research.ibm.com/ journal/rd/423/alt.pdf>. Acesso em: 5 set. 2003.

4. AMERICAN BOARD OF ORTHODONTICS. Specific instructions for candidates. Ottawa, 1990

5. BOCK, M. FilmeX Digital. Fotografe Melhor, São Paulo, n. 58, p. 30-41, 2001.

6. BRANCO, S.; AZZI, T. Entrada para o mundo das digitais. Fotografe Melhor, São Paulo, n. 65, p. 32-47, 2002.

7. GUIA prático digital: um mundo em evolução. Fotografe MeIhor, São Paulo, 2003, n. 3, p. 10-13, 2003.

8. HUTCHINSON, I.; IRELAND A. J.; STEPHENS, C. D. Digital cameras and orthodontics: an overview. Dent Update, Guildford, v. 26, p. 144-149, May 1999.

9. LOPES, A.; MACHADO, C.; VIEIRA, E.; GREGO, M. A hora do clique digital. Info Exame, São Paulo, n. 197, p. 52-78, ago. 2002.

10. MEGAPIXEL. The relationship between megapixels and printed image size. Disponível em: <http://www.megapixel. net/html/issueindex.php?lang=en>. Acesso em: 19 set. 2003.

11. POPULAR photography \& Imaging. Film vs. Digital, [S. I.], v. 67, no. 7, p. 76-83, July 2003.

12. RITTO, A. K. Imaging in orthodontics: present and future. J Clin Orthod, Boulder, v. 36, no. 11, p. 619-625, Nov. 2002.

13. SAEG 8. 0. Sistema de análises estatísticas. [S. I.]: UFV, 2000.

14. SANDLER, J.; MURRAY, A. Digital photography in orthodontics. J Orthod, Boulder, v. 28, p. 197-201, Sept. 2001.

15. STEWART, M. B. Management \& marketing. J Clin Orthod, Boulder, v. 29, no. 8, p. 509-515, Aug. 1995.

16. SWARTZ, M. L. Managing digital images. Am J Orthod Dentofacial Orthop, St. Louis, v. 118, no. 3, p. 354-358, Sept. 2000.
17. TRIGO, T. Equipamento fotográfico: teoria e prática. 2. ed. São Paulo: Senac, 2003.

18. VILANI, G. N.; LEITE, E.; ALMEIDA, J. M.; LOTTI, R. S.; PIMENTA, R. A.; LEITE, T. H. M. Fotografia convencional $X$ digital: um estudo comparativo preliminar. In: JORNADA ODONTOLÓGICA DA PUC MINAS, 13., 2003. Anais... Belo Horizonte: PUC, 2003. p. 29

19. WALL, S.; KAZAHAYA, K.; BECKER, S. S.; BECKER, D. G. Thirtyfive milimeter versus digital photography: comparison of photographic quality and clinical evaluation. Facial Plastic Surgery, New York, v. 15, no. 2, p. 101-109, 1999.

20. WILCOX, L. R.; GRIMWOOD, R. E. A comparative study of digital images versus 35 -milimeter images. Mil Med, Washington, D.C., v. 160 , no. 9, p. $470-472$, Sept. 1995. 